\title{
Impact of the educational technology use in undergraduate pharmacy teaching and learning - A systematic review
}

\author{
Chooi Yeng Lee (iD), Shaun Wen Huey Lee (iD) \\ School of Pharmacy, Monash University Malaysia, Malaysia
}

\author{
Keywords \\ Educational technology \\ Learning experience \\ Pharmacy teaching \\ Systematic review \\ Undergraduate \\ Correspondence \\ Chooi Yeng Lee \\ School of Pharmacy \\ Monash University Malaysia \\ Bandar Sunway \\ Subang Jaya \\ Selangor \\ Malaysia 47500 \\ chooi.yeng.lee@monash.edu
}

\begin{abstract}
Background: Educational technology has been increasingly used in recent years in pharmacy education. Its benefit on teaching and learning as well as its intention of use should be determined. Aims: To understand the technological approaches used in pharmacy education, and the impact of each approach on teaching and learning. Method: Four databases (PubMed, EMBASE, PsycINFO and ERIC) were searched to identify studies that described the use of technology in undergraduate pharmacy teaching and learning. Results: Thirty-four papers met the inclusion criteria. Majority of the studies (59\%) used simulation technologies adopting a situated learning approach to complement or enhance teaching and learning. Most of the studies reported change in two or more concepts of the Kirkpatrick's model, namely Reaction, Learning, and Behaviour, indicating improvement in learning experience, engagement, and performance. Conclusion: Educational technology with design features aligned with effective pedagogical theories seemed more likely to produce positive student outcomes.
\end{abstract}

\begin{abstract}
Introduction
Online synchronous and asynchronous learning, both in science, technology, engineering, and medicine (STEM) and non-STEM education, have been used in higher education for almost two decades. Asynchronous learning, in particular, is increasingly transforming the way education is being delivered. The development can be attributed to the change in the learning behaviour of the millennial generations, and more importantly, to the findings that blended learning improves learning effectiveness (Crouch, 2009; McLaughlin et al., 2015). The shift of the paradigm, as well as the increased demand of using innovative, advanced, and contemporary educational technology in higher education as a mode of teaching and learning, highlights the needs to ascertain that these technologies help to achieve the learning outcomes that were not achieved previously and that the technology is not developed just to satisfy the quest for its use.
\end{abstract}

A review by Salter and the authors (2014) on the effectiveness of e-learning in pharmacy education concluded that e-learning effectively increases knowledge and is a highly acceptable instructional format for students. But they also noted there was limited evidence that supports e-learning improves skills or professional practice, and there was no evidence that e-learning increases knowledge in the long term (Salter et al., 2014). Since the review in 2014, there has not been any update or similar systematic review conducted, suggesting a timely review of the subject is necessary. The lack of evidence of the benefit of e-learning on long term knowledge gain could be because this effect is difficult to demonstrate from relatively short interventional studies. However, examining the pedagogical theory or strategy adopted by researchers during the design of their e-learning tools, which was not done in the previous review, may lead the authors to the long term impact of an approach to learning. This paper aimed to review the current use of technology in pharmacy education, specifically on its relevance and effectiveness and attempted to address the limitations noted in Salter and the authors' (2014) review. With the increased abundance, variety, and access to synchronous and 
asynchronous learning tools, the focus of this paper was on technologies that were developed to enhance learning rather than studies that reported the use of e-learning only. The term technology-enhanced learning (TEL) is often utilised to describe a broad field of digital technologies used to support and mediate educational activities. In this review, the term TEL is used to describe totally digitally mediated activities and those that are blended with more traditional educational approaches, as detailed in the study inclusion criteria.

\section{Method}

\section{Data sources and search}

This review was conducted in accordance with the PRISMA guidelines. The following four databases, PubMed, EMBASE, PsycINFO, and ERIC, were searched from database inception to February 2019 for articles describing the use of technology to complement learning and teaching in the undergraduate pharmacy programme. The full electronic search strategy used was described in Appendix A. Search terms used include: blended, computer-assisted, computer-based, digital, electronic (E), electronic, mobile, information communication technology (ICT), information technology (IT), pharmacy, pharmacy education, and virtual.

\section{Study inclusion}

Articles were included if the studies meet the following criteria: 1) described an educational intervention to support and mediate educational activities; 2) used technologies to complement or enhance the learning experience, performance, or self-efficacy; and 3) were published between 2009 to 2019. Studies were excluded if they were non-research articles, discussed curriculum design, were conducted for distance learning programmes, or involved graduate pharmacists.

\section{Article selection, data extraction, and quality appraisal}

All records were exported into Endnote X8 (Clarivate Analytics, Philadelphia, PA, USA) and de-duplicated. Articles were screened by title and abstract by two independent reviewers, CYL and SWHL, for eligibility based upon the inclusion/exclusion criteria mentioned above. Any disagreement was resolved through a discussion between the two reviewers. Full text of relevant articles was retrieved and independently extracted using a data extraction form, which has been previously piloted for this review. Data extracted include: 1) the study characteristics such as the authors and the year of publication; 2) the description of the TEL tool; 3) participants; 4) assessment technique and measurement tools used; and 5) outcomes. A meta-narrative approach to synthesis was adopted since there was variation between studies in terms of the study design, intervention, outcomes, and study methods. The studies were described based on the technologies used and the key outcome measured in each study. According to Kirkpatrick's hierarchy model (Kirkpatrick, 1996), these were broadly themed and categorised to yield a concept map (Figure 1).

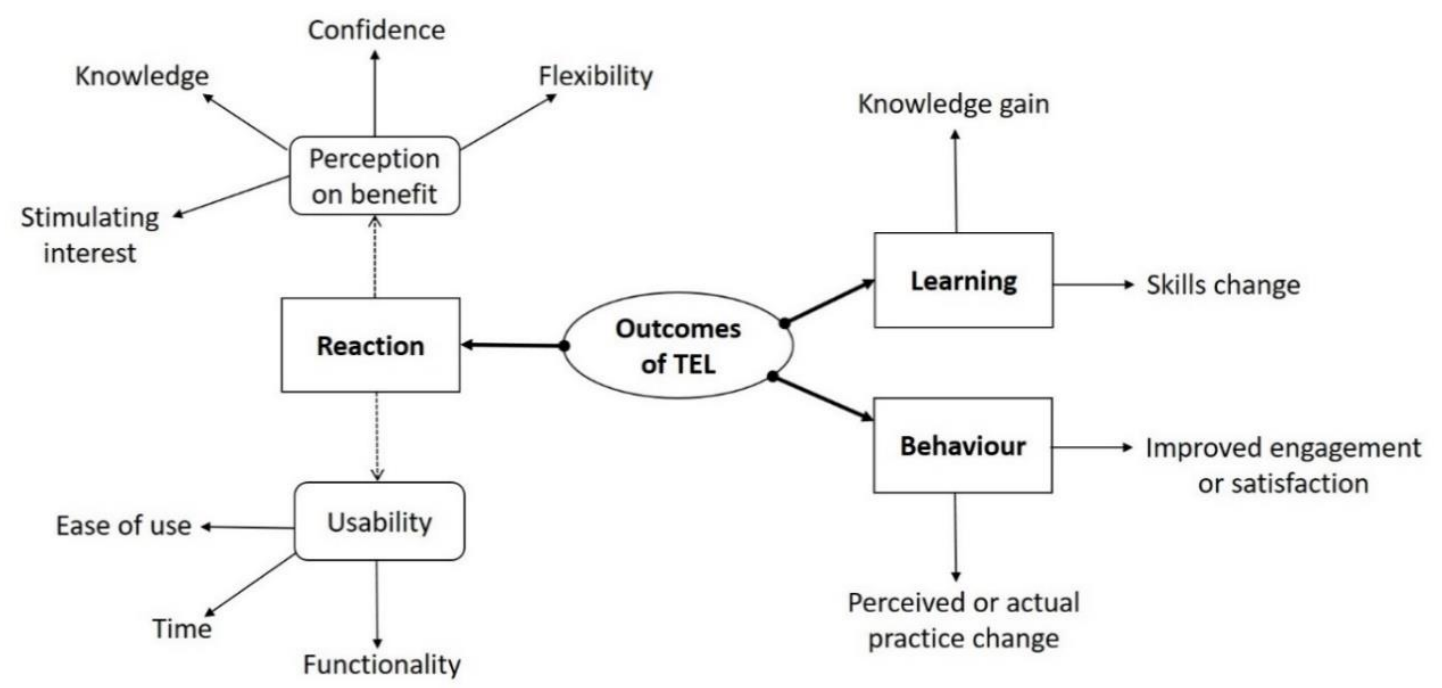

Figure 1: Categorisation of technology-enhanced learning (TEL) outcomes according to Kirkpatrick's hierarchy model

\section{Kirkpatrick's hierarchy model}

The model, first introduced in 1959 by Donald Kirkpatrick, aimed to clarify the meaning of evaluation and offer guidelines on how to get started and proceed. It consists of four levels of evaluation, which was described in the following order - 'reaction', 'learning', 
'behaviour', and 'results'. The model stood the test of time, as the content has remained unchanged when revisited in 1996.

'Reaction' is a measure of satisfaction to allow management to make decisions about training and to ensure that participants are motivated and interested in learning. 'Learning' is a measure of the knowledge acquired, skills improved, or attitudes changed due to training. 'Behaviour' is a measure of the extent to which participants change their on-the-job behaviour because of training. 'Results' is a measure of the final results that occur due to training (Kirkpatrick, 1996). Although the model was conceptualised for evaluating training programmes in the cooperate world, it shares similarity with the evaluation of a teaching and learning process in education. The purpose of evaluation, i.e. training, and participants of the training, can be referred to as the teaching and learning process, and students, respectively, while the management is the academics.

\section{Results}

\section{Characteristics of identified studies}

There were 1,105 papers identified from the database search, and 1,005 papers were excluded as they were either not related to the pharmacy programmes, nonresearch in nature or did not assess the learning outcomes. The full text of the remaining 100 papers was examined, and 66 papers, which did not meet all the inclusion criteria, were further excluded. Thirtyfour papers considered suitable for review were analysed for their TEL settings as well as the learning outcomes achieved (Figure 2).

The included studies were from the United States $(n=16)$, Europe $(n=9)$, Australia $(n=4)$, Asia $(n=3)$, Fiji $(n=1)$, and Brazil $(n=1)$ (Table I).
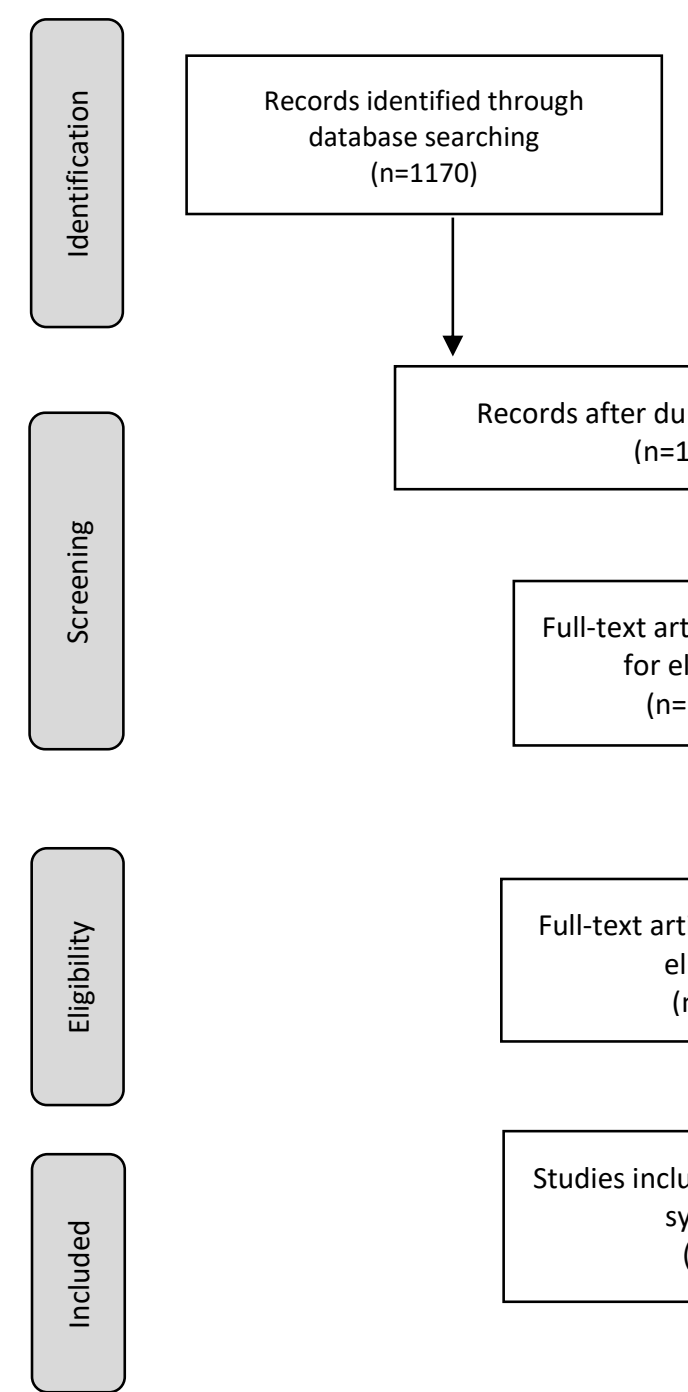
$(n=1170)$
Additional records identified through other sources $(n=1)$

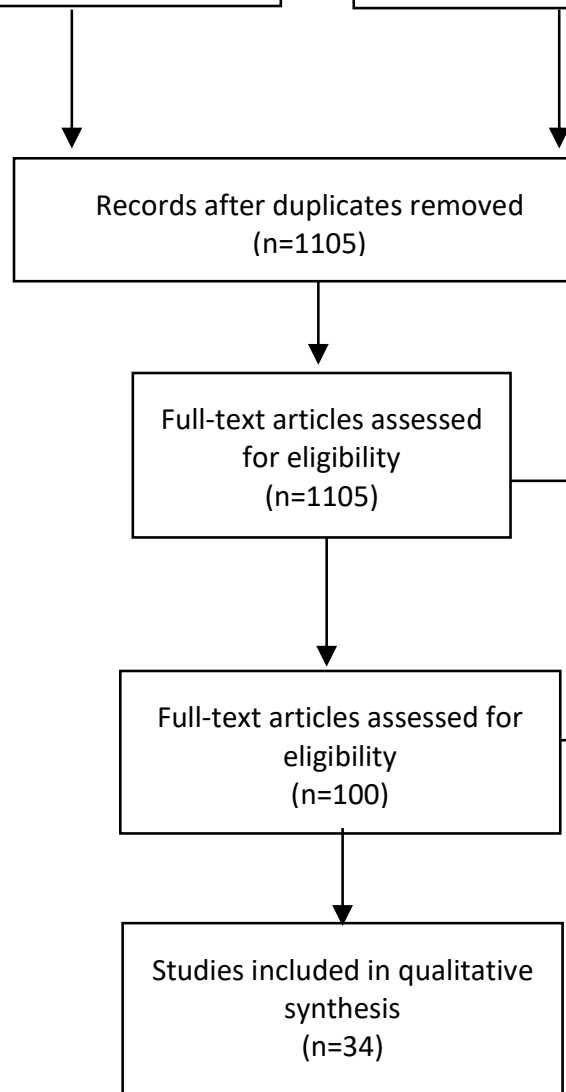

Records excluded $(n=1005)$

Figure 2: Study flow diagram 
Table I: Characteristics of the included studies

\begin{tabular}{|c|c|c|c|c|c|c|c|c|c|}
\hline \multirow{2}{*}{$\begin{array}{c}\text { First author, } \\
\text { Year } \\
\text { (Country) }\end{array}$} & \multirow{2}{*}{$\begin{array}{c}\text { Number of } \\
\text { participants, } \\
\text { Year of study } \\
\text { in Pharmacy } \\
\text { course }\end{array}$} & \multirow{2}{*}{$\begin{array}{l}\text { Topic delivered } \\
\text { by e-learning }\end{array}$} & \multirow{2}{*}{ E-learning intervention } & \multirow{2}{*}{$\begin{array}{l}\text { e-learning } \\
\text { setting }\end{array}$} & \multirow{2}{*}{$\begin{array}{c}\text { No of } \\
\text { session }\end{array}$} & \multirow{2}{*}{ Comparator } & \multicolumn{3}{|c|}{$\begin{array}{c}\text { Kirkpatrick's } \\
\text { hierarchy assessed }\end{array}$} \\
\hline & & & & & & & 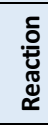 & 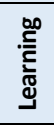 & 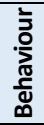 \\
\hline $\begin{array}{l}\text { Al-Dahir, } 2014 \\
\text { (USA) }\end{array}$ & $119,4^{\text {th }}$ & $\begin{array}{l}\text { Pharmacy } \\
\text { practice }\end{array}$ & $\begin{array}{l}\text { Virtual patient cases } \\
\text { using DecisionSim } \\
\text { software }\end{array}$ & Online & NR & PBL groups & $\mathrm{x}$ & & \\
\hline $\begin{array}{l}\text { Alsharif, } 2009 \\
\text { (USA) }\end{array}$ & $160,1^{\text {st }}-5^{\text {th }}$ & $\begin{array}{l}\text { Medicinal } \\
\text { chemistry }\end{array}$ & $\begin{array}{l}\text { Integrated pre-requisite } \\
\text { information } \\
\text { electronically using } \\
\text { SoftChalk }\end{array}$ & Online & NR & $\begin{array}{l}\text { No integration of } \\
\text { information }\end{array}$ & $\mathrm{x}$ & & \\
\hline $\begin{array}{l}\text { Ambroziak, } \\
2018 \text { (USA) }\end{array}$ & $85,1^{\text {st }}$ & $\begin{array}{l}\text { Pharmacy } \\
\text { practice }\end{array}$ & $\begin{array}{c}\text { Virtual dispensing, } \\
\text { MyDispense }\end{array}$ & Online & NR & NA & $\mathrm{x}$ & & $x$ \\
\hline $\begin{array}{l}\text { Barnett, } 2016 \\
\text { (USA) }\end{array}$ & $134,3^{\text {rd }}$ & $\begin{array}{l}\text { Pharmacy } \\
\text { practice }\end{array}$ & $\begin{array}{c}\text { Virtual patient case } \\
\text { using Case } \\
\text { Scenario/Critical Reader } \\
\text { Builder authoring tool }\end{array}$ & Online & NR & Paper case & $\mathrm{x}$ & $x$ & $\mathrm{x}$ \\
\hline $\begin{array}{l}\text { Benedict, } \\
2010 \text { (USA) }\end{array}$ & $107,3^{\text {rd }}$ & $\begin{array}{l}\text { Pharmacy } \\
\text { practice }\end{array}$ & $\begin{array}{l}\text { Virtual patient case } \\
\text { using DecisionSim } \\
\text { software }\end{array}$ & Online & 3 & NR & & $x$ & $x$ \\
\hline $\begin{array}{l}\text { Benedict, } \\
2013 \text { (USA) }\end{array}$ & $106,3^{\text {rd }}$ & $\begin{array}{l}\text { Pharmacy } \\
\text { practice }\end{array}$ & $\begin{array}{l}\text { Virtual patient case } \\
\text { using DecisionSim } \\
\text { software }\end{array}$ & Online & 3 & NR & $\mathrm{x}$ & & $x$ \\
\hline $\begin{array}{l}\text { Berger, } 2018 \\
\text { (Switzerland) }\end{array}$ & $70,5^{\text {th }}$ & $\begin{array}{l}\text { Pharmacy } \\
\text { practice }\end{array}$ & $\begin{array}{c}\text { Serious game } \\
\text { developed using an } \\
\text { authoring tool ITyStudio }\end{array}$ & Online & 1 & $\begin{array}{l}\text { Text-based } \\
\text { scenario }\end{array}$ & $\mathrm{x}$ & & \\
\hline $\begin{array}{l}\text { Bernaitis, } \\
2018 \\
\text { (Australia) }\end{array}$ & $28,4^{\text {th }}$ & $\begin{array}{c}\text { Oncology } \\
\text { pharmacotherapeu } \\
\text { tics (pharmacy } \\
\text { practice) }\end{array}$ & $\begin{array}{l}\text { Virtual patient case } \\
\text { using DecisionSim } \\
\text { software }\end{array}$ & Online & NR & $\begin{array}{l}\text { Without exposure } \\
\text { to DecisionSim }\end{array}$ & & $x$ & $x$ \\
\hline $\begin{array}{l}\text { Bindoff, } 2014 \\
\text { (Australia) }\end{array}$ & $33,3^{\text {rd }} \& 4^{\text {th }}$ & $\begin{array}{l}\text { Pharmacy } \\
\text { practice }\end{array}$ & $\begin{array}{c}\text { Simulation of a } \\
\text { community pharmacy } \\
\text { using Unity3D }\end{array}$ & Online & 2 & Paper-based & $\mathrm{x}$ & $\mathrm{x}$ & $x$ \\
\hline $\begin{array}{l}\text { Bryant, } 2017 \\
\text { (USA) }\end{array}$ & $67,1^{\text {st }}$ & $\begin{array}{l}\text { Basic health } \\
\text { sciences } \\
\text { laboratory }\end{array}$ & $\begin{array}{l}\text { Case studies developed } \\
\text { using iSolve for mobile } \\
\text { devices (iPad, iPhone, } \\
\text { smartphone \& tablet } \\
\text { computer) }\end{array}$ & Online & 1 & NA & $x$ & & \\
\hline $\begin{array}{l}\text { Cavaco, } 2012 \\
\text { (Portugal) }\end{array}$ & $194,4^{\text {th }} \& 5^{\text {th }}$ & $\begin{array}{l}\text { Pharmacy } \\
\text { practice }\end{array}$ & $\begin{array}{l}\text { Virtual patient } \\
\text { technology to simulate } \\
\text { and replace real-life } \\
\text { clinical scenario }\end{array}$ & Online & NR & $\begin{array}{c}\text { Without virtual } \\
\text { patient experience }\end{array}$ & $x$ & & \\
\hline $\begin{array}{l}\text { Coyne, } 2018 \\
\text { (USA) }\end{array}$ & $18,1^{\text {st }}$ & $\begin{array}{l}\text { Pharmacy } \\
\text { education }\end{array}$ & $\begin{array}{l}\text { Virtual reality (VR) as a } \\
\text { platform to provide } \\
\text { engaging elements for } \\
\text { team-based learning }\end{array}$ & VR & NR & None & $x$ & & $x$ \\
\hline $\begin{array}{l}\text { Ezeala, } 2013 \\
\text { (Fiji) }\end{array}$ & $42,2^{\text {nd }}$ & Pharmacology & $\begin{array}{l}\text { Simulated practical } \\
\text { sessions with } \\
\text { CyberPatient } 2007 \\
\text { software and Virtual } \\
\text { Organ Bath computer } \\
\text { software }\end{array}$ & In-class & 1 & NA & & $x$ & \\
\hline $\begin{array}{l}\text { Ferrone, } 2017 \\
\text { (USA) }\end{array}$ & $241,3^{\text {rd }}$ & $\begin{array}{c}\text { Dispensing } \\
\text { (practice skills) }\end{array}$ & $\begin{array}{c}\text { Virtual dispensing, } \\
\text { MyDispense }\end{array}$ & Online & 12 & NA & $x$ & & $x$ \\
\hline $\begin{array}{l}\text { Flowers, } 2010 \\
\text { (USA) }\end{array}$ & $79,4^{\text {th }}$ & $\begin{array}{l}\text { Pharmacy } \\
\text { practice }\end{array}$ & $\begin{array}{l}\text { Multimedia vignettes } \\
\text { on complex drug } \\
\text { administration } \\
\text { technique }\end{array}$ & Online & NR & $\begin{array}{l}\text { Students who did } \\
\text { not access the } \\
\text { vignettes }\end{array}$ & & $x$ & \\
\hline $\begin{array}{l}\text { Gustafsson, } \\
2017 \\
\text { (Sweden) }\end{array}$ & $42,4^{\text {th }}$ & $\begin{array}{l}\text { Pharmacy } \\
\text { practice }\end{array}$ & $\begin{array}{l}\text { 3D virtual world in } \\
\text { clinical pharmacy } \\
\text { teaching }\end{array}$ & Online & NR & None & $\mathrm{x}$ & & $x$ \\
\hline $\begin{array}{l}\text { Hall, } 2017 \\
\text { (Australia) }\end{array}$ & $24,3^{\text {rd }}$ & $\begin{array}{l}\text { Medicinal } \\
\text { chemistry }\end{array}$ & $\begin{array}{l}\text { 3D printed molecular } \\
\text { modelling tools }\end{array}$ & In-class & NR & Non-participants & & $x$ & $x$ \\
\hline
\end{tabular}




\begin{tabular}{|c|c|c|c|c|c|c|c|c|c|}
\hline \multirow{2}{*}{$\begin{array}{l}\text { First author, } \\
\text { Year } \\
\text { (Country) }\end{array}$} & \multirow{2}{*}{$\begin{array}{c}\text { Number of } \\
\text { participants, } \\
\text { Year of study } \\
\text { in Pharmacy } \\
\text { course }\end{array}$} & \multirow{2}{*}{$\begin{array}{l}\text { Topic delivered } \\
\text { by e-learning }\end{array}$} & \multirow{2}{*}{ E-learning intervention } & \multirow{2}{*}{$\begin{array}{l}\text { e-learning } \\
\text { setting }\end{array}$} & \multirow{2}{*}{$\begin{array}{l}\text { No of } \\
\text { session }\end{array}$} & \multirow{2}{*}{ Comparator } & \multicolumn{3}{|c|}{$\begin{array}{c}\text { Kirkpatrick's } \\
\text { hierarchy assessed }\end{array}$} \\
\hline & & & & & & & 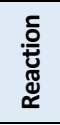 & 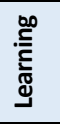 & 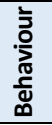 \\
\hline $\begin{array}{l}\text { Harrap, } 2016 \\
\text { (United } \\
\text { Kingdom) }\end{array}$ & $170, N R$ & $\begin{array}{l}\text { Pharmaceutical } \\
\text { calculation }\end{array}$ & Calculations e-package & Online & NR & None & $\mathrm{x}$ & $x$ & \\
\hline $\begin{array}{l}\text { Henriksen, } \\
2012 \\
\text { (Netherlands) }\end{array}$ & $56,1^{\text {st }}$ & $\begin{array}{l}\text { Medicinal } \\
\text { chemistry }\end{array}$ & $\begin{array}{l}\text { A learning management } \\
\text { system to allow } \\
\text { distance students to } \\
\text { hear and view students } \\
\text { on campus and } \\
\text { participate in workshop, } \\
\text { quizzes, and problem- } \\
\text { solving }\end{array}$ & Online & 1 & & $\mathrm{x}$ & & \\
\hline $\begin{array}{l}\text { Kurono, } 2015 \\
\quad \text { (Japan) }\end{array}$ & $470,1^{\text {st }}-4^{\text {th }}$ & $\begin{array}{l}\text { Communication } \\
\text { skills (practice } \\
\text { skills) }\end{array}$ & $\begin{array}{l}\text { DocCom - a } \\
\text { communication } \\
\text { learning tool }\end{array}$ & Online & NR & $\begin{array}{l}\text { before and after } \\
\text { intervention }\end{array}$ & $x$ & $x$ & \\
\hline $\begin{array}{l}\text { Lean, } 2018 \\
\text { (Malaysia) }\end{array}$ & $120,2^{\text {nd }}$ & $\begin{array}{l}\text { Pharmacy } \\
\text { practice }\end{array}$ & $\begin{array}{l}\text { Web-based online } \\
\text { learning module to } \\
\text { teach communication } \\
\text { skills }\end{array}$ & Online & NR & NA & & $x$ & $\mathrm{x}$ \\
\hline $\begin{array}{l}\text { Lee, } 2018 \\
\text { (Malaysia) }\end{array}$ & $30,2^{\text {nd }}$ & Pharmacology & $\begin{array}{l}\text { Articulate storyline } \\
\text { software for teaching } \\
\text { and gamification }\end{array}$ & Online & 1 & NA & $x$ & & $\mathrm{x}$ \\
\hline $\begin{array}{l}\text { Marriott, } \\
2012 \\
\text { (Australia) }\end{array}$ & $34,1^{\text {st }}-4^{\text {th }}$ & $\begin{array}{l}\text { Pharmacy } \\
\text { practice }\end{array}$ & $\begin{array}{l}\text { Resource platform } \\
\text { consisting of a fictional } \\
\text { community of people to } \\
\text { augment learning in an } \\
\text { undergraduate } \\
\text { pharmacy programme } \\
\text { and to refine patient } \\
\text { contact skills }\end{array}$ & Online & Variable & NA & $\mathrm{x}$ & & \\
\hline $\begin{array}{l}\text { Mattsson, } \\
2016 \\
\text { (Sweden) }\end{array}$ & $36,4^{\text {th }}$ & Drug formulation & Virtual tablet machine & Online & 1 & NA & $\mathrm{x}$ & & $\mathrm{x}$ \\
\hline $\begin{array}{l}\text { McLaughlin, } \\
2015 \text { (USA) }\end{array}$ & $95,2^{\text {nd }}$ & $\begin{array}{l}\text { Cardiovascular } \\
\text { pharmacotherapy }\end{array}$ & $\begin{array}{l}\text { Interactive online } \\
\text { module to replace } \\
\text { lecture }\end{array}$ & Online & NR & $\begin{array}{l}\text { Did not access all } \\
\text { segments of the } \\
\text { online module }\end{array}$ & & $x$ & $\mathrm{x}$ \\
\hline $\begin{array}{l}\text { Menendez, } \\
2015 \text { (Brazil) }\end{array}$ & $31, N R$ & $\begin{array}{l}\text { Pharmacy } \\
\text { practice }\end{array}$ & $\begin{array}{l}\text { A virtual patient system } \\
\text { to teach } \\
\text { communication skills }\end{array}$ & Online & NR & NA & $\mathrm{x}$ & & \\
\hline $\begin{array}{l}\text { Nazar, } 2018 \\
\text { (United } \\
\text { Kingdom) }\end{array}$ & $53,2^{\text {nd }}$ & Pharmacy law & $\begin{array}{l}\text { Video format of } \\
\text { illustrative tutorials, } \\
\text { where the audio talked } \\
\text { through the element of } \\
\text { pharmacy law, whilst a } \\
\text { digitalised hand- } \\
\text { illustrated visual aids to } \\
\text { accompany and support } \\
\text { that information }\end{array}$ & Online & 9 & NA & $\mathrm{x}$ & & $\mathrm{x}$ \\
\hline $\begin{array}{l}\text { Reinhold, } \\
2010 \text { (USA) }\end{array}$ & $27,2^{\text {nd }}$ & $\begin{array}{l}\text { Substance abuse } \\
\text { and drug } \\
\text { diversion }\end{array}$ & $\begin{array}{l}\text { Web-based educational } \\
\text { module }\end{array}$ & Online & NR & NA & $x$ & $\mathrm{x}$ & \\
\hline $\begin{array}{l}\text { Richardson, } \\
2013 \text { (United } \\
\text { Kingdom) }\end{array}$ & $40,2^{\text {nd }}$ & $\begin{array}{l}\text { Medicinal } \\
\text { chemistry }\end{array}$ & $\begin{array}{l}\text { Molecular visualisation } \\
\text { software PyMol. Images } \\
\text { projected using KAVE }\end{array}$ & In-class & 1 & $\begin{array}{l}\text { 3D group vs 2D } \\
\text { group }\end{array}$ & & $x$ & $x$ \\
\hline $\begin{array}{l}\text { Smith, } 2016 \\
\quad \text { (USA) }\end{array}$ & $102,3^{\text {rd }}$ & $\begin{array}{l}\text { Pharmacy } \\
\text { practice }\end{array}$ & $\begin{array}{l}\text { Virtual patient case } \\
\text { using DecisionSim } \\
\text { software }\end{array}$ & Online & 3 & & $\mathrm{x}$ & $x$ & $\mathrm{x}$ \\
\hline $\begin{array}{l}\text { Springer, } \\
2011 \text { (USA) }\end{array}$ & $45,5^{\text {th }}$ & $\begin{array}{l}\text { Pharmacogenom } \\
\text { ics }\end{array}$ & $\begin{array}{c}\text { Instructional } \\
\text { GeneScription software } \\
\text { system to teach a new } \\
\text { topic }\end{array}$ & Online & 8 & None & $\mathrm{x}$ & & \\
\hline $\begin{array}{l}\text { Taglieri, } 2017 \\
\quad \text { (USA) }\end{array}$ & $281,3^{\text {rd }}$ & $\begin{array}{l}\text { Pharmacy } \\
\text { practice }\end{array}$ & $\begin{array}{l}\text { Web-based Shadow } \\
\text { Health Digital Clinical }\end{array}$ & Online & NR & $\begin{array}{l}\text { VP before and after } \\
\text { mock clinic visits }\end{array}$ & $x$ & $\mathrm{x}$ & \\
\hline
\end{tabular}




\begin{tabular}{|c|c|c|c|c|c|c|c|c|c|}
\hline \multirow{2}{*}{$\begin{array}{c}\text { First author, } \\
\text { Year } \\
\text { (Country) }\end{array}$} & \multirow{2}{*}{$\begin{array}{c}\text { Number of } \\
\text { participants, } \\
\text { Year of study } \\
\text { in Pharmacy } \\
\text { course }\end{array}$} & \multirow{2}{*}{$\begin{array}{c}\text { Topic delivered } \\
\text { by e-learning }\end{array}$} & \multirow{2}{*}{ E-learning intervention } & \multirow{2}{*}{$\begin{array}{l}\text { e-learning } \\
\text { setting }\end{array}$} & \multirow{2}{*}{$\begin{array}{l}\text { No of } \\
\text { session }\end{array}$} & \multirow{2}{*}{ Comparator } & \multicolumn{3}{|c|}{$\begin{array}{c}\text { Kirkpatrick's } \\
\text { hierarchy assessed }\end{array}$} \\
\hline & & & & & & & 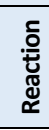 & 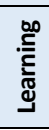 & 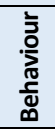 \\
\hline $\begin{array}{l}\text { Zlotos, } 2010 \\
\text { (Scotland) }\end{array}$ & $243,3^{\text {rd }}$ & $\begin{array}{l}\text { Pharmacy } \\
\text { practice }\end{array}$ & $\begin{array}{c}\text { Experience virtual } \\
\text { patient to teach clinical } \\
\text { skills } \\
\text { SCRIPT to compliment } \\
\text { classroom teaching of } \\
\text { competency-based } \\
\text { pharmacy practice }\end{array}$ & Online & NR & Non-user of SCRIPT & $\mathrm{x}$ & $x$ & \\
\hline $\begin{array}{l}\text { Zlotos, } 2015 \\
\text { (Scotland) }\end{array}$ & $433,3^{\text {rd }}$ & $\begin{array}{l}\text { Simulated } \\
\text { prescription } \\
\text { analysis } \\
\text { (pharmacy } \\
\text { practice) }\end{array}$ & $\begin{array}{l}\text { Computerised } \\
\text { randomised interactive } \\
\text { prescription tutor } \\
\text { (SCRIPT) }\end{array}$ & Online & NR & $\begin{array}{l}\text { Extent of } \\
\text { technology } \\
\text { integration }\end{array}$ & $x$ & & \\
\hline
\end{tabular}

In terms of the study design, eight were randomised controlled trials, 22 were quasi-experimental studies, three were case-control studies and one cross-sectional survey. The majority of the studies were from pharmacy practice $(n=20)$, followed by medicinal chemistry $(n=4)$, pharmacology $(n=3)$, and specific therapeutic areas such as pharmacogenetics, drug abuse, pharmaceutics, pharmacy law, pharmacy education, calculations and laboratory science. The total number of participants ranged from 18 to 470 , encompassed students from the first year to the fifth year of their study.

\section{Pharmacy practice}

Fourteen or $70 \%$ of the pharmacy practice studies described the use of virtual patient technology (Benedict, 2010; Cavaco \& Madeira, 2012; Benedict et al., 2013; Al-Dahir et al., 2014; Bindoff et al., 2014; Menendez et al., 2015; Barnett et al., 2016; Smith et al., 2016; Ferrone et al., 2017; Gustafsson et al., 2017; Taglieri et al., 2017; Ambroziak et al., 2018; Berger et al., 2018; Bernaitis et al., 2018). The remaining 30\% involved the use of simulated prescription (Zlotos et al., 2010; Zlotos et al., 2015), web-based multimedia vignettes (Flowers et al., 2010), learning module (Lean et al., 2018), communication tool (Kurono et al., 2015), and a resource platform consists of a fictional community of people (Marriott et al., 2012).

\section{Pharmacology and medicinal chemistry}

The software known as GeneScription that displays patient pharmacogenomics profile was used to enhance the knowledge and skills required in making clinical decisions (Springer et al., 2011). Other approaches used in pharmacology teaching included simulated patient and organ software for practical sessions (Ezeala et al., 2013) and interactive pharmacotherapy module for blending learning of the subject (McLaughlin et al., 2015; Lee et al., 2018). In medicinal chemistry, researchers created online learning modules to evaluate the appropriateness of distance learning of the subject (Alsharif \& Henriksen, 2009; Henriksen \& Roche, 2012) and used 3dimensional printing technology to teach drugreceptor interactions (Richardson et al., 2013; Hall et al., 2017).

\section{Other topics}

Web-based instructional modules were developed for the teaching of drug abuse and diversion (Reinhold et al., 2010) and pharmacy law (Nazar et al., 2019); virtual reality technology was tested for its effect on learning experience (Coyne et al., 2018); e-calculation tool to teach pharmaceutical calculation (Harrap et al., 2016); virtual tablet machine to teach drug formulation (Mattsson et al., 2016), and mobile devices for solving case studies of basic health sciences (Bryant \& Richard, 2017).

\section{Categorisation of papers based upon Kirkpatrick's model}

The papers reviewed fall in three of the four levels of Kirkpatrick's model, namely 'reaction', 'learning', and 'behaviour', with 22 or $65 \%$ of the studies demonstrated more than one level of Kirkpatrick's model. Specifically, 13 of the 16 studies, which have reported improved experience or engagement (81\%, Behaviour level), were also perceived to have benefits on knowledge (four studies, Reaction level) or have led to knowledge gain (nine studies, Learning level).

Overall, $32 \%$ of the studies reported students satisfaction with the TEL, perceiving benefits on knowledge (Springer et al., 2011; Cavaco \& Madeira, 
2012; Henriksen \& Roche, 2012; Marriott et al., 2012; Benedict et al., 2013; Kurono et al., 2015; Menendez et al., 2015; Mattsson et al., 2016; Gustafsson et al., 2017; Lee et al., 2018), 41\% reported positive outcome on knowledge gain (Benedict, 2010; Flowers et al., 2010; Reinhold et al., 2010; Zlotos et al., 2010; Ezeala et al., 2013; Richardson et al., 2013; Bindoff et al., 2014; McLaughlin et al., 2015; Barnett et al., 2016; Harrap et al., 2016; Smith et al., 2016; Hall et al., 2017; Bernaitis et al., 2018; Lean et al., 2018), which was determined either from measuring the difference in test scores before and after the intervention, or from examination performance, and $47 \%$ noted improved learning experience or engagement (Table II).

Table II: Outcomes of studies, themed against Kirkpatrick's four levels model

\begin{tabular}{|c|c|c|}
\hline \multicolumn{2}{|c|}{ Study outcomes } & Number of studies \\
\hline \multicolumn{3}{|c|}{ Reaction } \\
\hline \multicolumn{3}{|c|}{ - Perceived benefits on: } \\
\hline ○ & Knowledge & 11 \\
\hline o & Confidence & 6 \\
\hline o & Flexibility & 4 \\
\hline ○ & Stimulates interest & 10 \\
\hline ○ & Online discussion & 0 \\
\hline \multicolumn{3}{|c|}{ - Functionality: } \\
\hline$\circ$ & Technology & 2 \\
\hline ○ & Ease of use & 8 \\
\hline ○ & Time & 3 \\
\hline \multicolumn{3}{|c|}{ Learning } \\
\hline - $\mathrm{Knc}$ & vledge gain & 14 \\
\hline - $\quad \operatorname{Incr}$ & ased confidence & 0 \\
\hline - $\quad$ Skil & change & 4 \\
\hline \multicolumn{3}{|c|}{ Behaviour } \\
\hline \multicolumn{2}{|c|}{$\begin{array}{l}\text { - Improved engagement or } \\
\text { satisfaction }\end{array}$} & 16 \\
\hline \multicolumn{2}{|c|}{$\begin{array}{l}\text { Perceived or actual practice } \\
\text { change }\end{array}$} & 3 \\
\hline \multicolumn{2}{|c|}{ - Willingness to change practice } & 0 \\
\hline
\end{tabular}

\section{Discussion}

Pharmacy practice was the discipline that has used educational technology the most, and the majority of the studies adopted virtual or simulation technologies. A virtual learning environment is a situated learning approach as it takes learners through the processes to yield the desired knowledge (Rutto, 2017). The virtual patient programmes reviewed enabled students to identify the patient's problem and make a recommendation, and provided feedback to students based on the recommendation that students made. Simulation technologies have also been used in and benefited other pharmacy disciplines. In medicinal chemistry and pharmacology, simulation, which allows visualisation rather than imagination of the molecular structures, has enhanced students understanding of drug-receptor interactions (Richardson et al., 2013).
This is expected because simulation offers opportunities for active and engaging learning (Cain \& Fox, 2009). Its benefits, therefore, should not be limited to the teaching and learning of complex clinical concepts or professional skills. The majority of the simulation studies reported perceived benefits on knowledge or practice skills, an example of Reaction level based on Kirkpatrick's model, increased knowledge gain (Learning level) and improved learning experience or engagement (Behaviour level). The benefits of simulation are unequivocal, but other TEL tools have generated positive outcomes as well.

In a study by Lee and authors (2018), students who were introduced to an interactive learning software for the learning of pharmacology perceived improvement in their understanding of concepts and principles of the subject and found the TEL interesting and engaging. Four other studies reviewed, which also did not use simulation technologies (Richardson et al., 2013; McLaughlin et al., 2015; Hall et al., 2017; Lean et al., 2018), reported improvement in learning and engagement. Self-directed learning (SDL) is defined by Gibbons (2002) as an increase in knowledge, skill, accomplishment, or personal development brought about by an individual's own effort in any circumstances at any time. A simulation that fosters clinical decision-making skills promotes SDL, and SDL encourages life-long learning (Benedict et al., 2013). In line with the definition of SDL, it is anticipated that if a TEL is successful in achieving its intended outcomes, the knowledge change may be long-term, and the tool may not necessarily be a simulation technology. On this note, whether or not a TEL will encourage SDL may depend on how the technology has changed students' Reaction, Learning and Behaviour, or Reaction and Behaviour, or Learning and Behaviour.

Compared to the educational technologies reviewed previously (Salter et al., 2014), which involved mostly learning management platforms, more advanced and more varieties of technologies are used in pharmacy education. They ranged from highly interactive simulation technologies for patient counselling, tablet production, devices demonstration, and experimental process to technologies that aimed to provide immersive learning experience such as virtual reality.

Studies that focused on improving knowledge of specific subjects or skills have generally been successful. These studies include tablet formulation, pharmacy law, pharmaceutics calculation, pharmacokinetics, and pharmacodynamics. They used strategies consistent with transformed practice and constructivist learning theories. Transformed practice is a pedagogy that enables learners to put learned knowledge into practice or transfer meaning-making to work in a new social context (The New London Group, 1996). This 
pedagogy was exemplified by Springer and the authors (2011) in their study. They developed a GeneScription software to allow the screening of drug-gene interaction during the prescription filling process, thereby allowing students to make decisions of altering the dose of the drug based on the pharmacogenomics information provided by the system. Meanwhile, constructivist learning theories emphasise the active involvement of the learners during the knowledge construction process (Rutto, 2017). Active learning strategies such as providing an opportunity for interaction with others or an environment are used to engage students in the learning process (Jonassen, 1999). Situated learning, a learning theory categorised under constructivism, is demonstrated more often than other learning theories in the present review. Effective implementation of the strategies of constructivism is able to improve students knowledge and interest (Reaction) and engagement and motivation (Behaviour) in the learning of a multidisciplinary subject such as pharmacotherapy (Lee, 2020). This may explain, in part, the success of the studies in demonstrating outcomes described by Kirkpatrick's model.

The present study was limited by the inclusion criteria, where only studies that have measured learning outcomes were included. Research that evaluated the usability of new technology but without measuring students' reaction, learning, behaviour, and performance from the use of the technology was excluded. As the main intention of the present review was to understand if the recent used of technologies has enhanced teaching and learning in pharmacy education, this study excluded approaches that were reviewed before as well as those that have been commonly used, such as recorded lectures and forums. There may be a possibility of overlooking some relevant studies due to the limitation of the search databases and that the search terms were not exhaustive enough to capture all the studies.

\section{Conclusion}

This study showed that there were benefits of the rapid advancement of current technologies as there was an improvement in students learning experiences, knowledge, and professional skills. Simulation technologies were used in all pharmacy disciplines and were mostly successful in achieving the above outcomes. Other TEL approaches have also improved students' performance through increasing the interactive features of the design (for better engagement), addressing the knowledge gaps (between classroom and practice), and increasing access to resources (for better guidance). Regardless of the disciplines, the constructivist learning strategy and the transformed practice strategy are among the pedagogical theories indicated in the above studies.

\section{Conflicts of interest}

The authors declare no conflict of interest.

\section{References}

Al-Dahir, S., Bryant, K., Kennedy, K. B., \& Robinson, D. S. (2014). Online virtual-patient cases versus traditional problem-based learning in advanced pharmacy practice experience. American Journal of Pharmaceutical Education, 78, 76. https://doi.org/10.5688/ajpe78476

Alsharif, N. Z., \& Henriksen, B. (2009). Electronic integration of prerequisite course content. American Journal of Pharmaceutical Education, 73, 150. https://doi.org/10.5688/aj7308150

Ambroziak, K., Ibrahim, N., Marshall, V. D., \& Kelling, S. E. (2018). Virtual simulation to personalize student learning in a required pharmacy course. Currents in Pharmacy Teaching and Learning, 10, 750-756. https://doi.org/10.1016/j.cptl.2018.03.017

Barnett, S. G., Gallimore, C. E., Pitterle, M., \& Morrill, J. (2016). Impact of a paper vs virtual simulated patient case on student-perceived confidence and engagement. American Journal of Pharmaceutical Education, 80, 16. https://doi.org/10.5688/ajpe80116

Benedict, N. (2010). Virtual patients and problem-based learning in advanced therapeutics. American Journal of Pharmaceutical Education, 74, 143. https://doi.org/10.5688/aj7408143

Benedict, N., Schonder, K., \& McGee, J. (2013). Promotion of self-directed learning using virtual patient case. American Joumal of Pharmaceutical Education, 77, 151. https://doi.org/10.5688/ajpe777151

Berger, J., Bawab, N., De Mooij, J., Widmer, D. S., Szilas, N., De Vriese, C., et al. (2018). An open randomized controlled study comparing an online text-based scenario and a serious game by Belgian and Swiss pharmacy students. Currents in Pharmacy Teaching and Learning, 10, 267-276. https://doi.org/10.1016/j.cptl.2017.11.002

Bernaitis, N., Baumann-Birkbeck, L., Alcorn, S., Powell, M., Arora, D., \& Anoopkumar-Dukie, S. (2018). Simulated patient cases using DecisionSim ${ }^{\mathrm{TM}}$ improves student performance and satisfaction in pharmacotherapeutics education. Currents in Pharmacy Teaching and Learning, 10, 730-735. https://doi.org/10.1016/j.cptl.2018.03.020

Bindoff, I., Ling, T., Bereznicki, L., Westbury, J., Chalmers, L., Peterson, G., et al. (2014). Acomputer simulation of community pharmacy practice for educational use. American Journal of Pharmaceutical Education, 78, 168. https://doi.org/10.5688/ajpe789168

Boeker, M., Andel, P., Vach, W., \& Frankenschmidt, A. (2013). Game-based e-learning is more effective than a conventional instructional method: a randomized controlled trial with third-year medical students. PLOS ONE, 8, e82328. https://doi.org/10.1371/journal.pone.0082328 
Bryant, J. E., \& Richard, C. A. H. (2017). Pharmacy students' use and perceptions of Apple mobile devices incorporated into a basic health science laboratory. Currents in Pharmacy Teaching and Learning, 9, 78-93. https://doi.org/10.1016/j.cptl.2016.08.044

Cain, J., \& Fox, B. I. (2009). Web 2.0 and pharmacy education. American Journal of Pharmaceutical Education, 73, 120. https://doi.org/10.5688/aj7307120

Cavaco, A. M., \& Madeira, F. (2012). European pharmacy students' experience with virtual patient technology. American Journal of Pharmaceutical Education, 76, 106. https://doi.org/10.5688/ajpe766106

Coyne, L., Takemoto, J. K., Parmentier, B. L., Merritt, T., \& Sharpton, R. A. (2018). Exploring virtual reality as a platform for distance team-based learning. Currents in Pharmacy Teaching and Learning, 10, 1384-1390. https://doi.org/10.1016/j.cptl.2018.07.005

Crouch, M. A. (2009). An advanced cardiovascular pharmacotherapy course blending online and face to-face instruction. American Journal of Pharmacy Education, 73, 51. https://doi.org/10.5688/aj730351

Ezeala, C. C., Ram, A. A., \& Vulakouvaki, N. (2013). Learning gain of pharmacy students after introducing guided inquiry learning with computer simulation in a pharmacology class in Fiji. Journal of Educational Evaluation for Health Professions, 10, 9. https://doi.org/10.3352/jeehp.2013.10.9

Ferrone, M., Kebodeaux, C., Fitzgerald, J., \& Holle, L. (2017). Implementation of a virtual dispensing simulator to support US pharmacy education. Currents in Pharmacy Teaching and Learning, 9, 511-520. https://doi.org/10.1016/j.cptl.2017.03.018

Flowers, S. K., Vanderbush, R. E., Hastings, J. K., \& West, D. (2010). Web-based multimedia vignettes in advanced community pharmacy practice experiences. American Journal of Pharmacy Education, 74, 39. https://doi.org/10.5688/aj740339

Gustafsson, M., Englund, C., \& Gallego, G. (2017). The description and evaluation of virtual worlds in clinical pharmacy education in Northern Sweden. Currents in Pharmacy Teaching and Learning, 9, 887-892. https://doi.org/10.1016/j.cptl.2017.06.002

Hall, S., Grant, G., Arora, D., Karaksha, A., McFarland, A., Lohning, A., et al. (2017). A pilot study assessing the value of 3D printed molecular modeling tools for pharmacy student education. Currents in Pharmacy Teaching and Learning, 9, 723-728. https://doi.org/10.1016/j.cptl.2017.03.029

Harrap, N., Usman, S., McLaughlin, C., Orwell, S., Harris, S. A., Ling, V., et al. (2016). Using a blended approach to support calculation skills of pharmacy students. Pharmacy Education, 16, 189-198

Hatala, R., Cook, D. A., Zendejas, B., Hamstra, S. J., \& Brydges, R. (2014). Feedback for simulation-based procedural skills training: a meta-analysis and critical narrative synthesis. Advance in Health Sciences Education: Theory and Practice, 19, 251-272. https://doi.org/10.1007/s10459-013-9462-8

Hattie, J., \& Timperley, H. (2007). The power of feedback. Review of Educational Research, 77, 81-112. https://doi.org/10.3102/003465430298487

Henriksen, B., \& Roche, V. (2012). Creation of medicinal chemistry learning communities through enhanced technology and interdisciplinary collaboration. American Journal of Pharmaceutical Education, 76, 158. https://doi.org/10.5688/ajpe768158

Jonassen, D. (1999). Designing for constructivist learning environments. In C. M. Reigeluth (Ed.), Instructional-design theories and models: A new paradigm of instructional theory. New Jersey: Lawrence Erlbaum Associates

Jones, J. S., Tincher, L., Odeng-Out, E., \& Herdman, M. (2015). An educational board game to assist PharmD students in learning autonomic nervous system pharmacology. American Journal of Pharmaceutical Education, 79, 114. https://doi.org/10.5688/ajpe798114

Kirkpatrick, D. (1996). Great ideas revisited: revisiting Kirkpatrick's four-level model. Training and Development, 50, 54-60

Kurono, S., Hanya, M., Kamei, H., \& Hasegawa, Y. (2015). Evaluation of a web-based curriculum resource "DocCom" by pharmacy students' self-assessment. Pharmacy Education, 15, 99-104

Lean, Q. Y., Ming, L. C., Wong, Y. Y., Neoh, C. F., Farooqui, M., \& Muhsain, S. N. F. (2018). Validation of online learning in pharmacy education: effectiveness and student insight. Pharmacy Education, 18, 135-142

Lee, C. Y. (2020). How to improve the effectiveness of blended learning of pharmacology and pharmacotherapy? A case study in pharmacy program. Technology, Knowledge and Learning, 25, 977-988. https://doi.org/10.1007/s10758020-09447-5

Lee, C. Y., \& Sen, A. (2018). Students voice in their learning: incorporating students' expectations in learning design of elearning of pharmacotherapy. International Journal of Learning and Teaching, 4, 203-208

Lee, C. Y., White, P. J., \& Malone, D. T. (2018). Online educational games improve the learning of cardiac pharmacology in undergraduate pharmacy teaching. Pharmacy Education, 18, 298-302

Marriott, J., Styles, K., \& McDowell, J. (2012). The Pharmville community: a curriculum resource platform integrating context and theory. American Journal of Pharmaceutical Education, 76, 178. https://doi.org/10.5688/ajpe769178

Mattsson, S., Sjöström, H., \& Englund, C. (2016). Using a virtual tablet machine to improve student understanding of the complex processes involved in tablet manufacturing. American Journal of Pharmaceutical Education, 80, 87. https://doi.org/10.5688/ajpe80587

McLaughlin, J. E., Gharkholonarehe, N., Khanova, J., Deyo, Z. M., \& Rodgers, J. E. (2015). The impact of blended learning on student performance in a cardiovascular pharmacotherapy course. American Journal of Pharmaceutical Education, 79, 24. https://doi.org/10.5688/ajpe79224

Menendez, E., Balisa-Rocha, B., Jabbur-Lopes, M., Costa, W., Nascimento, J. R., Dósea, M., et al. (2015). Using a virtual patient system for the teaching of pharmaceutical care. International Journal of Medical Informatics, 84, 640-646. https://doi.org/10.1016/j.ijmedinf.2015.05.015 
Nazar, H., Omer, U., Nazar, Z., \& Husband, A. (2018). A study to investigate the impact of a blended learning teaching approach to teach pharmacy law. International Journal of Pharmacy Practice. https://doi.org/10.1111/ijpp.12503

Reinhold, J., Pontiggia, L., Angeles, M., \& Earl, G. (2010). Webbased instruction on substance abuse and drug diversion. American Journal of Pharmaceutical Education, 74, 57. https://doi.org/10.5688/aj740457

Richardson, A., Bracegirdle, L., McLachlan, S. I. H., \& Chapman, S. R. (2013). Use of a three-dimensional virtual environment to teach drug-receptor interactions. American Journal of Pharmaceutical Education, 77, 11. https://doi.org/10.5688/ajpe77111

Salter, S. M., Karia, A., Sanfilippo, F. M., \& Clifford, R. M. (2014). Effectiveness of e-learning in pharmacy education. American Journal of Pharmaceutical Education, 78, 83. https://doi.org/10.5688/ajpe78483

Smith, M. A., Siemianowski, L. A., \& Benedict, N. (2016). Virtual patient case sharing across two schools of pharmacy. American Journal of Pharmaceutical Education, 80, 153. https://doi.org/10.5688/ajpe809153

Springer, J. A., lannotti, N. V., Kane, M. D., Haynes, K., \& Sprague, J. E. (2011). Pharmacogenomics training using an instructional software system. American Journal of Pharmaceutical Education, 75, 32. https://doi.org/10.5688/ajpe75232

Taglieri, C. A., Crosby, S. J., Zimmerman, K., Schneider, T., \& Patel, D. K. (2017). Evaluation of the use of a virtual patient on student competence and confidence in performing simulated clinic visits. American Journal of Pharmaceutical Education, 81, 87. https://doi.org/10.5688/ajpe81587

The New London Group. (1996). A pedagogy of multiliteracies: Designing social future. Harvard Educational Review, 66, 60-92. https://doi.org/10.17763/haer.66.1.17370n67v22j160u

Zlotos, L., Kayne, L., Thompson, I., Kane, K. A., \& Boyter, A. (2010). A web-based tool for teaching pharmacy practice competency. American Journal of Pharmaceutical Education, 74, 27. https://doi.org/10.5688/aj740227

Zlotos, L., Thompson, I. D., \& Boyter, A. C. (2015). Integration of an online simulated prescription analysis into undergraduate pharmacy teaching using supplemental and replacement models. American Journal of Pharmaceutical Education, 79, 37. https://doi.org/10.5688/ajpe79337

\section{Appendix A. Sample search strategy}

Sample search strategy used in the present systematic review in PubMed. This was adapted for the databases ERIC, EMBASE via Ovid and PsychINFO.

1. electronic

2. blended

3. distance

4. computer assisted

5. computer based

6. digital

7. electronic

8. mobile

9. information communication technology

10. ICT

11. information technology

12. IT

13. virtual

14. \#1 OR \#2 OR \#3 OR \#4 OR \#5 OR \#6 OR \#7 OR \#8 OR \#9 OR \#10 OR \#11 OR \#12 OR \#13

15. education

16. education program

17. learning program

18. knowledge

19. \#15 OR \#16 OR \#17 OR \#18

20. pharmacy

21. pharmacist*

22. \#20 OR \#21

23. \#14 AND \#19

24. \#22 AND \#23 